\title{
Development of a Program to Educate Physician Assistant Students on Performance of a Comprehensive Breast Exam
}

\author{
Pamela B. Jaffey \\ Nova Southeastern University, pjaffey@nova.edu \\ Diana Cherkiss \\ Nova Southeastern University, diana.cherkiss@nova.edu \\ Mara Sanchez \\ Duke University, mara.sanchez@duke.edu \\ Patrick Hardigan \\ Nova Southeastern University, patrick@nova.edu
}

Follow this and additional works at: https://nsuworks.nova.edu/ijahsp

Part of the Curriculum and Instruction Commons, Educational Methods Commons, and the Medicine and Health Sciences Commons

\section{Recommended Citation}

Jaffey PB, Cherkiss D, Sanchez M, Hardigan P. Development of a Program to Educate Physician Assistant Students on Performance of a Comprehensive Breast Exam. The Internet Journal of Allied Health Sciences and Practice. 2019 Jan 01;17(4), Article 3.

This Manuscript is brought to you for free and open access by the College of Health Care Sciences at NSUWorks. It has been accepted for inclusion in Internet Journal of Allied Health Sciences and Practice by an authorized editor of NSUWorks. For more information, please contact nsuworks@nova.edu. 


\title{
Development of a Program to Educate Physician Assistant Students on Performance of a Comprehensive Breast Exam
}

\begin{abstract}
ABSTRACT

Purpose: The purpose of this study was to develop a new program to educate physician assistant (PA) students on the performance of a comprehensive breast exam and to test its effectiveness as compared to the previous program. Methods: The preexisting educational program for the performance of a clinical breast exam was evaluated during the term prior to the start of clinical rotations for the class of 2015. Baseline data were obtained from this control group by evaluation of student competency using a skills examination, utilizing a Nasco Advanced Breast Exam Simulator, and using a female standardized patient. An innovative program educating the PA class of 2016 on the performance and interpretation of a comprehensive breast exam was instituted, incorporating a new palpation technique, new models, and simulated patients. Both classes were directly compared in terms of their breast examination skills at 5 months post-training by administering the same skills examination. Nested, mixed, generalized, and linear models were created to look for differences between the two classes. Each model was adjusted by covariates to account for variation in student ability. Results: The PA class of 2016 showed statistically better performance in breast inspection, lymph node palpation, breast palpation, breast mass detection, and documentation of findings compared to the PA class of 2015. Conclusion: The new educational program on the clinical breast exam using a well-organized laboratory session with subsequent skills testing was found to be very efficacious.
\end{abstract}

\section{Author Bio(s)}

Pamela B. Jaffey, M.D. is an Associate Professor of physician assistant education in the Dr. Pallavi Patel College of Health Care Sciences at Nova Southeastern University in Fort Lauderdale, FL. Diana E. Cherkiss, MMS, MPH, PA-C is an Assistant Professor of physician assistant education in the Dr. Pallavi Patel College of Health Care Sciences at Nova Southeastern University in Fort Lauderdale, FL. She currently practices family medicine with a focus on functional and regenerative medicine. Mara R. Sanchez, MMS, PA-C is an Assistant Professor in Community and Family Medicine at Duke University's Physician Assistant Program in North Carolina. She is also a registered Dietitian. Patrick C. Hardigan, Ph. D., M.B.A is the Director of the Statistical Consulting Center at Nova Southeastern University in Fort Lauderdale, FL. He is also a Professor of Public Health in the Dr. Kiran C. Patel College of Osteopathic Medicine. 


\title{
IIVAHSP \\ The Internet Journal of Allied Health Sciences and Practice \\ Dedicated to allied health professional practice and education
}

Vol. 17 No. 4 ISSN 1540-580X

\section{Development of a Program to Educate Physician Assistant Students on Performance of a Comprehensive Breast Exam}

\author{
Pamela B. Jaffey \\ Diana Cherkiss \\ Mara Sanchez \\ Patrick Hardigan \\ Nova Southeastern University \\ United States
}

\begin{abstract}
Purpose: The purpose of this study was to develop a new program to educate physician assistant (PA) students on the performance of a comprehensive breast exam and to test its effectiveness as compared to the previous program. Methods: The preexisting educational program for the performance of a clinical breast exam was evaluated during the term prior to the start of clinical rotations for the class of 2015. Baseline data were obtained from this control group by evaluation of student competency using a skills examination, which incorporated a Nasco Advanced Breast Exam Simulator and a female standardized patient. An innovative program educating the PA class of 2016 on the performance and interpretation of a comprehensive breast exam was instituted, incorporating a new palpation technique, new models, and simulated patients. Both classes were directly compared in terms of their breast examination skills at 5 months post-training by administering the same skills examination. Nested, mixed, generalized, and linear models were created to look for differences between the two classes. Each model was adjusted by covariates to account for variation in student ability. Results: The PA class of 2016 showed statistically better performance in breast inspection, lymph node palpation, breast palpation, breast mass detection, and documentation of findings compared to the PA class of 2015. Conclusion: The new educational program on the clinical breast exam using a well-organized laboratory session with subsequent skills testing was found to be very efficacious.
\end{abstract}

Keywords: physical exam, curriculum development, innovations 


\section{INTRODUCTION}

Breast cancer is the second leading cause of cancer death in women in developed countries. ${ }^{1}$ Mammograms are useful for breast cancer screening, but miss $8-17 \%$ of breast cancer cases. ${ }^{2}$ Several studies have shown that the sensitivity of combined clinical breast exam (CBE) and mammography is higher than the sensitivity of mammography alone in the detection of breast

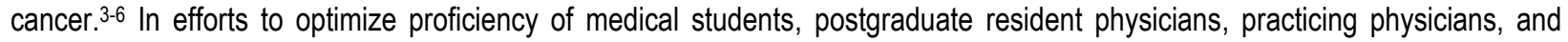
nurse practitioners in the performance of a $\mathrm{CBE}$, numerous training programs have been developed and reported. Reviews of many training programs have been conducted by MacDonald et al and Dilaveri et al. 7,8 There have not been any publications to date by a physician assistant (PA) program which describes a program directed toward educating PA students on the performance of a CBE. These findings indicate that a need exists for a report of a program that trains PA students to perform an efficacious comprehensive breast exam.

An educational program for PA students enrolled in the Nova Southeastern University Physician Assistant Program in Fort Lauderdale, Florida, on the performance of a CBE was developed and compared with the effectiveness of the program used in the previous cohort. In the new program, students were taught breast exam techniques allowing more thorough palpation of the breasts as described by the American Cancer Society (ACS). ${ }^{9}$ The ACS has defined breast exam boundaries in the shape of a pentagon, including the mid-axillary line, across the inframammary ridge (at the fifth or sixth rib), up the lateral edge of the sternum, across the clavicle, and back to the mid-axilla. In the previous program, the breast exam boundaries included the outer conical area of the breast with an additional small amount of breast tissue extending from the upper outer conical boundary to the tail of Spence.

Thoroughness in palpation technique was also promoted in the new program by teaching the "vertical strip" pattern of breast palpation rather than the "concentric circle" pattern taught in the previous program. Its increased comprehensiveness compared to the concentric circle method was initially demonstrated by Saunders at al. ${ }^{10}$ These investigators found that female participants, who were educated on self-breast exam by both methods, palpated the conical area of their breast more completely with the vertical strip method than by the concentric circle method according to a numbered grid projected on their chest. ${ }^{10}$ The vertical strip pattern was subsequently found by Steiner et al to be a factor correlating strongly with successful detection by medical residents of a $3 \mathrm{~mm}$ breast mass in a silicone model. ${ }^{11}$ Furthermore, Benincasa et al demonstrated that training primary care physicians to perform a vertical strip search pattern contributed to much greater accuracy in the detection of 5 masses in a silicone breast model as compared to a previous attempt before the vertical strip training. ${ }^{12}$

While the preexisting program utilized static breast models with fixed breast masses for students to palpate, the new program utilized the Nasco Life/Form Advanced Breast Exam Simulator. ${ }^{12,13}$ This model allows instructors to create a variety of clinical exam scenarios by inserting breast masses of different sizes, shapes, consistency, depth, and mobility at different locations within the breast. Additional models that had not been used in the previous program were MammaCare silicone breast models. ${ }^{14}$ These allow students to detect masses as small as $3 \mathrm{~mm}$ within simulated breast tissue. Another innovation was the use of a standardized skills checklist according to the breast exam protocol described by the ACS. ${ }^{9}$ Finally, a skills examination was arranged based on the skills checklist using the Nasco Life/Form Advanced Breast Exam Simulator and a standardized patient.

\section{METHODS}

The preexisting and new educational programs on the CBE were incorporated into the physical diagnosis course. The PA class of 2015 received the preexisting training while the PA class of 2016 received the new educational program. The laboratory session in both the previous and new programs included a demonstration of the breast exam on a male volunteer. There was also a second session in which students performed a breast exam on a female standardized patient. The primary laboratory sessions of both the previous and new programs, however, had major differences. In the earlier program, static breast models which did not have adjustable masses were used to train students during palpation. ${ }^{12}$ The models utilized with the previous cohort lacked the ability to palpate different breast textures, as what could be palpable in female human breast tissue. During the preexisting lab, students examined the models independently and without faculty feedback. Students also practiced breast inspection and lymph node palpation on each other without faculty supervision. Finally, while there was training on documentation of exam findings, there was no formal evaluation.

The new educational program on the CBE utilized new, dynamic models, which were incorporated into a more extensive laboratory session. The Nasco Life/Form advanced breast exam simulator was used to create a variety of breast findings allowing students to contrast breast tissue without a mass as compared to breast tissue with masses of different shapes, sizes, mobility, consistency, and depth in the tissue. ${ }^{13}$ Students were taught to palpate the models according to the method described by the ACS and followed a standardized skills checklist. Two MammaCare silicone breast models were also utilized in the lab. ${ }^{14}$ 
One model was clear in color to provide visualization as students palpated masses of different sizes, consistency, and depth in the tissue. A portion of the clear model had masses within that simulated physiologic nodularity. The masses were as small as 3 $\mathrm{mm}$. The other model was opaque with masses for students to practice palpation techniques. Students were given round stickers to place on locations of the breast model where they determined a mass was present. They were able to assess their accuracy looking at the clear side on the underside of the model. Students were given significant faculty supervision and feedback while examining the models.

The laboratory session was organized into four stations utilizing direct faculty supervision: breast inspection and lymph node palpation where students practiced on each other, documentation of breast masses using a static Nasco torso model, palpation of the Nasco Life/Form advanced breast exam simulator, and palpation of the MammaCare silicone breast models. A second lab followed during which time the students performed a CBE on female standardized patients under direct faculty supervision.

After students completed the laboratory session and examination of the female standardized patients, they were administered a skills assessment examination involving breast inspection and lymph node palpation on a female standardized patient and palpation of the breasts using a Nasco Life/Form advanced breast exam simulator. The students then documented their findings on breast palpation on a diagram of the breasts, as well as in written format. Two faculty observers scored each student according to a checklist based upon the techniques published by the ACS. Students were required to obtain a total test score of $70 \%$ or above to pass the skills assessment. After the exam, the only feedback that students were given was whether they had passed the exam or not. They were not made aware of any of the findings in the breast simulator. Two students did not receive a passing score, and their techniques were remediated.

Both the PA class of 2015 and class of 2016 were administered the same skills examination 5 months after the breast examination training they had received, which was shortly prior to the start of clinical rotations. The exam was in the same format as the one used to assess the class of 2016 immediately after their training program; however, there were some important differences. The skills exam was incorporated into a completely different course, Health Promotion and Disease Prevention, rather than in the Physical Diagnosis course, as the students had already completed the Physical Diagnosis series of courses in the curriculum. Students were informed that they were going to be performing a cancer screening assessment as a requirement for the course but would not receive a grade. Both classes of 2015 and 2016 were not informed of the specific screening test they were going to perform; therefore, students in both classes of 2015 and 2016 most likely did not prepare for the skills exam.

The categories scored on the skills exam were the following: breast inspection, lymph node palpation, breast palpation, mass detection, and documentation of findings. A total score for breast palpation was determined, as well as a separate score that excluded the vertical strip palpation technique, the technique that had not been taught to the class of 2015 students. (The class of 2015 students had been taught the concentric circle palpation technique). By excluding the score associated with the vertical strip technique, the breast palpation score could be directly compared between the 2 classes. The documentation score was based upon the ability to document the mass size and mass location for two separate masses. The total exam score was determined by adding the scores for breast inspection, lymph node palpation, breast palpation without the vertical strip technique, and documentation.

To observe differences between the classes for the continuous scores, five nested, mixed, general linear models were created. The outcome variables were (1) total exam score, (2) inspection score, (3) lymph node palpation score, (4) breast palpation score, and (5) breast palpation score without vertical strip technique. To look for differences between the classes for the categorical exam scores (correct vs incorrect), four nested, mixed, generalized linear models were created. The outcome variables were (1) documentation of size, (2) documentation of location, (3) finding one of two masses (4) finding two of two masses. For both comparisons, the covariates were verbal reasoning GRE score, quantitative reasoning GRE score, analytic writing GRE score, cumulative GPA prior to entering the PA program, and current GPA while enrolled in the PA program. The fixed effects were grouped (2015 vs. 2016). The random effects were student and faculty evaluator. Students were nested within the faculty to control for the variation in faculty evaluators.

Descriptive statistics were calculated for all study variables. This included the mean and standard deviation for continuous measures and counts. Percentages were used for categorical variables. The statistical package R 3.2.2 was used to create and test all models. ${ }^{15} \mathrm{~A} p$-value of $p<0.05$ was used to test statistical significance.

(c) The Internet Journal of Allied Health Sciences and Practice, 2019 


\section{RESULTS}

The final sample included 70 and 71 students from the classes of 2015 and 2016, respectively. The average age of the 2016 cohort ( $M=25.3, S D=5.8)$ was very similar to the 2015 class $(M=23.9, S D=4.5)$. Correspondingly, gender composition was comparable as the class of 2015 was comprised of $86 \%$ females and $76 \%$ females in 2016 . The descriptive statistics for the covariates are found in Table 1. The descriptive statistics for continuous outcome variables total score, inspection, lymph node palpation, and breast palpation with and without the vertical strip search pattern) are depicted on Table 2 . Additionally, the results of pairwise comparisons are found on Table 3. Significant improvement was found across all measures for the 2016 cohort, including total score, inspection, lymph node palpation, breast palpation with and without the vertical strip search pattern, the ability to document mass size, mass location, and the ability to find both one and two masses.

Table 1. Descriptive Statistics for Study Co-Variates

\begin{tabular}{ccccccc}
\hline \multirow{3}{*}{2015} & Variable & N & Mean & SD & Min & Max \\
\cline { 2 - 7 } & vgre & 70 & 55.91 & 17.92 & 21.00 & 93.00 \\
& qgre & 70 & 56.80 & 15.24 & 26.00 & 94.00 \\
& agre & 70 & 53.51 & 17.92 & 6.00 & 92.00 \\
& cumgpa & 70 & 3.63 & 0.20 & 3.09 & 4.00 \\
& pagpa & 70 & 3.34 & 0.28 & 2.74 & 3.84 \\
\cline { 2 - 7 } 2016 & Variable & N & Mean & SD & Min & Max \\
\cline { 2 - 7 } & vgre & 71 & 59.49 & 19.11 & 10.00 & 95.00 \\
& qgre & 71 & 51.63 & 17.35 & 0.57 & 87.00 \\
& agre & 71 & 55.46 & 21.27 & 5.00 & 96.00 \\
& cumgpa & 71 & 3.62 & 0.22 & 2.94 & 4.00 \\
& pagpa & 71 & 3.31 & 0.34 & 2.63 & 3.97 \\
\hline
\end{tabular}

Abbreviations: Verbal Graduate Record Exam (VGRE), Quantitative Graduate Record Exam (QGRE), Analytical Graduate Record Exam (AGRE), Cumulative Grade Point Average (CumGPA), Physician Assistant Grade Point Average (PAGPA)

Table 2. Descriptive Statistics for Continuous Outcome Variables

\begin{tabular}{clrrrr}
\hline \multirow{2}{*}{2015} & Variable & SD & Min & Max \\
\cline { 2 - 5 } $\mathrm{N}=70$ & Total Score & 22.03 & 6.95 & 8.00 & 39.00 \\
& Inspection Score & 3.85 & 1.81 & 0.00 & 6.00 \\
& Lymph Node Palpation Score & 8.29 & 4.42 & 0.00 & 16.00 \\
& Breast Palpation Score With Vertical Strip Pattern & 11.86 & 2.92 & 6.00 & 19.00 \\
& Breast Palpation Score Without Vertical Strip Pattern & 5.86 & 2.92 & 0.00 & 13.00 \\
\cline { 2 - 6 } 2016 & $\mathrm{M}$ & $\mathrm{SD}$ & $\mathrm{Min}$ & Max \\
\cline { 2 - 6 } $\mathrm{N}=71$ & Variable & 36.32 & 4.67 & 21.00 & 46.00 \\
& Total Score & 4.78 & 1.80 & 0.00 & 6.00 \\
& Inspection Score & 11.86 & 3.18 & 0.00 & 16.00 \\
& Lymph Node Palpation Score & 19.05 & 1.86 & 14.00 & 24.00 \\
& Breast Palpation Score With Vertical Strip Pattern & 13.00 & 2.01 & 3.00 & 18.00 \\
\hline
\end{tabular}


Table 3 Results of pairwise comparisons

\begin{tabular}{cccccccc}
\hline Measure & Year & & Year & Difference & $\begin{array}{c}\text { Lower } \\
95 \% \mathrm{Cl}\end{array}$ & $\begin{array}{c}\text { Upper } \\
95 \% \mathrm{Cl}\end{array}$ & $\mathrm{P}$-Value \\
\hline Total Score & 2016 & Vs. & 2015 & 14.22 & 12.92 & 15.52 & $\mathrm{P}<0.001$ \\
Inspection & 2016 & Vs. & 2015 & 0.94 & 0.54 & 1.32 & $\mathrm{P}<0.001$ \\
Lymph Node Palpation & 2016 & Vs. & 2015 & 3.50 & 2.70 & 4.40 & $\mathrm{P}<0.001$ \\
Breast Palpation/VS* & 2016 & Vs. & 2015 & 7.00 & 6.40 & 7.60 & $\mathrm{P}<0.001$ \\
Breast Palpation/NVS** & 2016 & Vs. & 2015 & 3.50 & 2.65 & 4.39 & $\mathrm{P}<0.001$ \\
Document Size & 2016 & Vs. & 2015 & $81.5 \%$ & $80.4 \%$ & $82.6 \%$ & $\mathrm{P}<0.001$ \\
Document Location & 2016 & Vs. & 2015 & $65.9 \%$ & $64.7 \%$ & $67.1 \%$ & $\mathrm{P}<0.001$ \\
Detect One of Two Masses & 2016 & Vs. & 2015 & $65.9 \%$ & $64.7 \%$ & $67.1 \%$ & $\mathrm{P}<0.001$ \\
Detect Two of Two Masses & 2016 & Vs. & 2015 & $16.3 \%$ & $14.7 \%$ & $17.8 \%$ & $\mathrm{P}<0.001$ \\
\hline
\end{tabular}

${ }^{*}$ With Vertical Strip ** Without Vertical Strip

\section{DISCUSSION}

The results indicate that the new program in our study designed to teach PA students a comprehensive breast exam is more effective than the previous program taught one year prior. Learners acquire knowledge best when exposed to a variety of teaching modalities. ${ }^{27}$ The class of 2016 performed better than the class of 2015 in all categories tested on the skills examination. This was most likely due to the different stations that allowed students to experience multiple learning modalities in addition to a standardized patient experience. The visual, aural, read/write, and kinesthetic (VARK) learning style model teaches us that students learn in different ways. ${ }^{27}$ Even if a learner may have a preferred way of learning, educating students with a combination of these four modalities can enhance student knowledge and skills. The categories we assessed were breast inspection, lymph node palpation, breast palpation with and without the vertical strip pattern, and medical documentation.

The increased competency in breast palpation demonstrated by the class of 2016 was most likely responsible for their significantly greater success in breast mass detection as compared to the class of 2015. The documentation skills of the class of 2016 were also superior to those of the class of 2015. The fine documentation skills of the class of 2016 can be attributed to the increased instruction on documentation during the breast exam lecture given to the class of 2016, as compared to the lecture given to the class of 2015. In addition, while documentation practice was included in the laboratory session of the class of 2016, it was not included in the 2015 laboratory session.

This is the first program for the instruction of PA students on performance of a comprehensive breast exam to be reported to our knowledge. The results of the skills assessment exam demonstrated that it is efficacious in instructing students on breast inspection, lymph node palpation, breast palpation, detection of breast masses, and documentation of findings. This program has combined components that contribute to its usefulness: a thorough, well-organized laboratory session with beneficial breast models and direct faculty supervision, a female standardized patient lab, a skills checklist, and skills testing. The development of a checklist based on the protocol published by the ACS promoted student learning and objective evaluation of their examination techniques. The skills testing helped motivate the students to master the techniques.

The use of silicone breast models has been reported in various studies.711,16-18 These models have the advantage of simulating breast tissue and breast masses as small as $3 \mathrm{~mm}$, so we have utilized them in our program. ${ }^{14}$ They do not, however, simulate a 
female torso and have static breast masses. The Nasco Life/Form advanced breast exam simulator, which is used in our program, is a dynamic model that simulates a female torso. ${ }^{13}$

There are multiple reports which stress the importance of a CBE for breast cancer screening. ${ }^{19-23}$ However, this is not universally accepted.1,24 Performance of a high-quality CBE in the diagnosis of breast cancer when a woman presents to a clinician with a breast complaint is agreed on. ${ }^{25,26}$ The goal of our program is to prepare students to perform a CBE which is useful for both breast cancer screening and diagnosis.

\section{Limitations}

There are limitations to this research. The first involves the inability to prevent students initially evaluated on their performance of a breast exam from disseminating information to other students related to the suspected mass locations, as a holding room was not utilized. However, the stronger performance of the class of 2016 students in every category of the breast exam as compared to the class of 2015 students suggests that this potential occurrence did not invalidate the examination process. Another limitation was the lack of close tactile resemblance of the Nasco Life/Form advanced breast exam simulator to normal breast tissue. This resulted in greater difficulty to palpate the simulator. The simulator did allow for a more objective evaluation of student breast palpation skills as compared to a female standardized patient. It reproduced a torso and allowed different scenarios of normal breast tissue and breast masses to be created. In addition, students had the opportunity to palpate the breasts of female standardized patients.

\section{Recommendations for Future Research}

Further research in other physician assistant programs regarding implementation and outcomes of a CBE protocol would be appropriate to increase the current knowledge base. In addition, we recognize that all subjects were enrolled in a single PA program, which limits generalizability to other PA programs. Further research needs to be done using a larger number of students from different programs. The effectiveness of the new program, however, can be useful for other PA programs looking to develop their curricula on $\mathrm{CBE}$.

\section{CONCLUSION}

A new protocol for the instruction of PA students on the performance of a comprehensive breast exam has been created and validated by its effectiveness as compared to the previous protocol in the PA program at Nova Southeastern University in Fort Lauderdale, Florida. We note that all participants were enrolled in a single PA program, which limits generalizability to other PA programs. Nevertheless, it is hoped that the more enriched laboratory experience with enhanced breast models and increased direct faculty supervision, as well as the skills testing format, can be useful to other PA programs in their efforts to educate future PA students on the CBE. 


\section{References}

1. Lauby-Secretan B, Scoccianti C, Loomis D, et al. Breast-cancer screening-viewpoint of the IARC working group. N Engl J Med. 2015;372(24):2353-2358.

2. Goodson III WH, Hunt TK, Plotnik JN, Moore II DH. Optimization of clinical breast examination. Am J Med. 2010;123(4):329334. doi: 10.1016/j.amjmed.2009.08.023

3. Chiarelli AM, Majpruz V, Brown $P$, Theriault M, Shumak R, Mai V. The contribution of clinical breast examination to the accuracy of breast screening. J Natl Cancer Inst. 2009;101(18):1236-1243. doi: 10.1093/jnci/djp241

4. Oestreicher N, Lehman CD, Seger DJ, Buist DS, White E. The incremental contribution of clinical breast examination to invasive cancer detection in a mammography screening program. Am J Roentgenol. 2005:184:428-32.

5. Bobo, JK, Lee, NC, Thames SF. Findings from 752,081 clinical breast examinations reported to a national screening program from 1995 through 1998. J Natl Cancer Inst. 2000;92(12):971-976.

6. Jatoi I. Screening clinical breast examination. Surg Clin N Am. 2003;83:789-801.

7. McDonald S, Saslow D, Alciati MH. Performance and reporting of clinical breast examination: a review of the literature. $C A$ Cancer J Clin. 2004;54(6):345-361.

8. Dilaveri CA, Szostek JH, Wang AT, Cook DA. Simulation training for breast and pelvic physical examination: a systematic review and meta-analysis. BJOG. 2013;120:1171-1182. doi: 10.1111/1471-0528.102289

9. Saslow D, Hannan J, Osuch J, et al. Clinical breast examination: practical recommendations for optimizing performance and reporting. CA Cancer J Clin. 2004;54(6):327-344.

10. Saunders KJ, Pilgrim CA, Pennypacker HS. Increased proficiency of search in breast self-examination. Cancer. 1986;58:2531-2537.

11. Steiner E, Austin DF, Prouser NC. Detection and description of small breast masses by residents trained using a standardized clinical breast exam curriculum. J Gen Intern Med. 2007;23(2):129-34. doi: 10.1007/s11606-007-0444-5

12. 3B Scientific GmbH. 2005. L510912336. https://www.a3bs.com/

13. Nasco Life/form Advanced breast exam simulator. http://www.enasco.com/product/LF00980U/.

14. MammaCare $\mathbb{R}$ Learning System MLS-1, MammaCare Foundation, Gainesville FI. 32601

15. R Core Team (2012). R: A language and environment for statistical computing. R Foundation for Statistical Computing, Vienna, Austria. ISBN 3-900051-07-0, URL http://www.R-project.org/

16. Pugh CM, Salud LH. Fear of missing a lesion: use of simulated breast models to decrease student anxiety when learning clinical breast examinations. Am J Surg. 2007;193:766-770. doi: 10.1016/j.amsurg.2006.12.033

17. Pilgrim C, Lannon C, Harris RP, Cogburn W, Fletcher SW. Improving clinical breast examination training in a medical school: a randomized control trial. J Gen Intern Med. 1993;8:685-688.

18. Benincasa TA, King ES, Rimer BK, et al. Results of an office-based training program in clinical breast examination for primary care physicians. J Cancer Educ. 1996;11:25-31.

19. Goodson III WH, Grissom NA, Moore II, DH, Dirbas FM. Streamlining clinical breast examination. J Natl Cancer Inst. 2005;97(19):1476-1477. doi: 10.1093/jnci/dji309 
20. Miller AB, Wall C, Baines CJ, Sun P, To T, Narod SA. Twenty five year follow-up for breast cancer incidence and mortality of the Canadian national breast screening study: randomised screening trial. BMJ. 2014;348:g366. doi: 10.1136/b,j.g366

21. Ravi C, Rodrigues G. Accuracy of clinical examination of breast lumps in detecting malignancy: a retrospective study. Indian J Surg Oncol. 2012;3(2):154-157. doi:10.1007/s13193-012-0151-5

22. Haakinson DJ, Stucky $\mathrm{CH}$, Dueck AC, et al. A significant number of women present with palpable breast cancer even with a normal mammogram within 1 year. Am J Surg. 2010;200:712-718. doi: 10.1016/j.amsurg.2010.08.005

23. American College of Obstetricians and Gynecologists. Well-woman visit. Committee Opinion No. 534. Obstet Gynecol 2012;120:421-4.

24. Oeffinger KC, Fontham ET, Etzioni R, et al. Breast cancer screening for women at average risk: 2015 guideline update from the American Cancer Society. JAMA. 2015;314(15):1599-1614. doi: 10.001/jama.2015.12783

25. Bryan T, Snyder E. The clinical breast exam: a skill that should not be abandoned. J Gen Intern Med. 2013;28:179-722. doi: 10.1007/s11606-013-2373-9

26. Barton MB, Elmore JG, Fletcher SW. Breast symptoms among women enrolled in a health maintenance organization: frequency, evaluation, and outcome. Ann Int Med. 1999;130:651-657.

27. Davis BG. Part VI: Enhancing Students' Learning and Motivation. In: Tools for Teaching. 2nd ed. San Francisco, CA: JosseyBass; 2009: 259-276. 
